\title{
O IMPACTO DA PANDEMIA DO NOVO CORONAVÍRUS NA QUALIDADE DE VIDA DE ESTUDANTES DE ENFERMAGEM
}

\section{THE IMPACT OF THE SARS-COV 2 PANDEMIC ON THE QUALITY OF LIFE OF NURSING STUDENTS}

\section{LA CALIDAD DE VIDA DE LOS ESTUDIANTES DE ENFERMERÍA EN EL CONTEXTO PANDÉMICO POR SARS-COV 2}

Tangriane Hainiski Ramos ${ }^{1}$, Edivane Pedrolo ${ }^{2}$, Leni de Lima Santana ${ }^{2}$, Nadine de Biagi Souza Ziesemer ${ }^{1}$, Rafael Haeffner ${ }^{3}$, Telma Pelaes de Carvalho ${ }^{4}$

\section{RESUMO}

Objetivo: Identificar o impacto da pandemia do coronavírus SARS-CoV2, na qualidade de vida de estudantes do curso técnico em enfermagem, quanto ao período de suspensão do calendário acadêmico. Método: Estudo transversal desenvolvido mediante formulário on-line, com alunos de uma instituição pública. Utilizou-se questionário composto por questões sociodemográficas e pelo The World Health Organization Quality of Life (WHOQOL-bref). Resultados: Foram incluídos 55 participantes. 63,6\% avaliaram sua qualidade de vida como boa e $47,3 \%$ estão satisfeitos com sua saúde. Na estratificação dos domínios do WHOQOL-bref, o "Físico" obteve o maior escore, 67,9\%, enquanto o "Psicológico" teve o menor escore, 58,2\%. O domínio "Psicológico" foi afetado significativamente pela situação conjugal $(p=0,0344)$, trabalho $(p=0,0392)$ e sustento da casa $(p=0,0196)$. Já a situação conjugal ( $p=$ $0,0025)$ e os filhos $(p=0,0212)$ tiveram relação significativa com a autoavaliação da qualidade de vida. Conclusão: Identificou-se que as variáveis relacionadas à situação conjugal, filhos, trabalho e sustento da casa alteram de forma significativa a qualidade de vida, durante a pandemia, dos estudantes investigados. Verificou-se também que, apesar da autoavaliação quanto à qualidade de vida $\mathrm{e}$ saúde serem boas, a dimensão psicológica é a mais afetada.

Descritores: Qualidade de vida; Estudantes de Enfermagem; Isolamento social; Infecções por Coronavírus; Pandemias.

\section{ABSTRACT}

Objective: To identify the impact of the SARS-CoV2 Coronavirus pandemic on the quality of life of nursing students during the suspension of the academic calendar. Method: A cross-sectional study developed using an online form, with students from a public institution. A questionnaire composed of sociodemographic questions and The World Health Organization Quality of Life (WHOQOLbref) instrument. Results: 55 students participated. $63.6 \%$ rated their quality of life as good and $47.3 \%$ said they were satisfied with their health. In the stratification of the WHOQOL-bref domains, the "Physical" obtained the highest score, 67.9\%, while "Psychological" had the lowest score, $58.2 \%$. The "Psychological" domain was significantly affected by marital status $(p=0.0344)$, work $(p=0.0392)$ and household income $(p=0.0196)$. The marital status $(p=0.0025)$ and the children $(p=0.0212)$ had a significant relationship with the self-assessment of quality of life. Conclusion: The variables related to marital status, children, work and household support significantly alter the quality of life, during the pandemic, of the students investigated. It was also found that, although the self-evaluation regarding their quality of life and health is good, the psychological dimension is the most affected.

Descriptors: Quality of Life; Students, Nursing; Social Isolation; Coronavirus Infection; Pandemics.

\section{RESUMEN}

Objetivo: Identificar el impacto de la pandemia del SARS-CoV2 en la calidad de vida de los estudiantes de enfermería durante la suspensión del calendario académico. Método: Estudio transversal desarrollado con estudiantes de una institución pública utilizando formularios electrónicos, sociodemográfico y la Calidad de Vida de la Organización Mundial de la Salud (WHOQOL-bref). Resultados: Participaron 55 estudiantes, de los cuales el $63,6 \%$ calificó su calidad de vida como buena y el $47,3 \%$ dijo estar satisfecho con su salud. En la estratificación de los dominios del WHOQOL-bref, "Físico" obtuvo la puntuación más alta, 67,9\%, mientras que "Psicológico" obtuvo la puntuación más baja, 58,2\%. El dominio "Psicológico" se vio afectado significativamente por el estado civil ( $p=0,0344)$, el trabajo $(p=0,0392)$ y los ingresos familiares $(p=0,0196)$. El estado civil $(p=0,0025)$ y los hijos $(p=0,0212)$ tuvieron una relación significativa con la autoevaluación de la calidad de vida. Conclusión: La situación conyugal, tener hijos, trabajar y mantener el hogar interfirió con la calidad de vida de los estudiantes investigados durante la pandemia. También se encontró que, si bien la autoevaluación en cuanto a calidad de vida y salud es buena, la dimensión psicológica es la más afectada.

Descriptores: Calidad de Vida; Estudiantes de Enfermería; Aislamiento Social; Infecciones por Coronavirus; Pandemias.

'Doutorado em Distúrbios da Comunicação pela Universidade Tuiuti do Paraná. Docente do Instituto Federal de Educação, Ciência e Tecnologia do Paraná. ${ }^{2}$ Doutorado em Enfermagem pela Universidade Federal do Paraná. Docente do Instituto Federal de Educação, Ciência e Tecnologia do Paraná. ${ }^{3}$ Doutorado em Epidemiologia pela Universidade de São Paulo. Enfermeiro da Prefeitura Municipal de São José dos Pinhais. ${ }^{4}$ Pós-Doutorado em Distúrbios da Comunicação pela Universidade Tuiuti do Paraná. Docente do Instituto Federal de Educação, Ciência e Tecnologia do Paraná.

Como citar este artigo:

Ramos TH, Pedrolo E, Santana LL, et al. Novo Coronavírus: O impacto da pandemia na qualidade de vida de estudantes de enfermagem. Revista de Enfermagem do Centro-Oeste Mineiro. 2020;10:e4042. [Access ]; Available in: DOI: 


\section{INTRODUÇÃO}

A chegada do novo Coronavírus - SARS-CoV2 e o consequente avanço da Doença (Corona Virus Disease - COVID-19), no Brasil, culminou com a tomada de decisão, por parte de autoridades governamentais de implementar $\mathrm{o}$ distanciamento social como principal medida para reduzir o contágio pelo vírus, uma vez que, até o momento, não há nenhuma terapêutica ou vacina específica para a doença. Em virtude do distanciamento social, uma série de atividades econômicas, sociais e educacionais foram suspensas, acompanhando os modelos de enfrentamento internacionais que incluem 0 fechamento de escolas, escritórios, repartições públicas e o cancelamento de outras atividades que resultem em aglomerações de pessoas ${ }^{(1)}$.

Quanto à essa nova realidade social, há mudanças nas rotinas e no modo de viver das pessoas, que têm seu direito de locomoção e de contato com as demais restringido. Essas medidas, ainda que necessárias para o enfrentamento da pandemia, em virtude do risco de contágio, são responsáveis por inúmeros impactos pessoais, tanto de ordem psicológica, como o estresse, a ansiedade e a depressão ${ }^{(2)}$, quanto de ordem social e física, como o isolamento social e o agravamento de doenças crônicas $^{(3-4)}$. As consequências psicológicas relacionam-se com receios em relação ao vírus ou à infecção, à frustração, à redução de rendimentos, à informação inadequada e à estigmatização social ${ }^{(2)}$.

No contexto acadêmico, mudanças na forma como são realizadas as atividades pedagógicas, em decorrência da implementação de um ensino remoto/on-line, imposto pelo distanciamento social, além das incertezas e dos impactos negativos na progressão acadêmica podem contribuir para a ocorrência de perturbações psicológicas ${ }^{(2-4)}$. As novas modalidades de ensino e os impactos na vida dos discentes têm sido um dilema vivenciado por professores e outros profissionais de instituições de ensino, uma vez que, ao tomar decisões que envolvam a continuidade do processo de ensinoaprendizagem, precisam pensar na manutenção da qualidade da educação ofertada, bem como na forma como elas impactarão na vida da comunidade interna, ao mesmo tempo em que precisam garantir a manutenção da saúde física e mental de todos os envolvidos.

Outro aspecto a se considerar sobre o impacto da pandemia na vida acadêmica se refere às desigualdades vivenciadas pelos estudantes, no desenvolvimento de atividades escolares no ambiente domiciliar, pela distinção nas condições de moradia, de recursos tecnológicos e mesmo de papéis sociais assumidos por homens $e$ mulheres ${ }^{(5)}$.

No que se refere à formação profissional do técnico em enfermagem, vale ressaltar que ela está diretamente relacionada ao cuidado, no qual toda a gama de conhecimento científico se encontra diretamente pautado e fundamentado na prática profissional, pois é dela que se dão interações entre alunos, comunidade e profissionais $^{(6)}$, necessidades que 0 ensino remoto/on-line não conseguem suprir. Portanto, além dos aspectos supracitados, relacionados à pandemia, apresentam-se outros advindos deste contexto desafiador do ensino nesta nova modalidade, principalmente, para uma profissão como a técnica em enfermagem, cujo desenvolvimento de habilidades técnicas requer grande carga horária de atividades práticas, o que certamente impacta na vida de educadores e educandos.

Todos esses aspectos podem influenciar diretamente na qualidade de vida (QV) das pessoas, sendo que a intensidade dessas consequências depende de características pessoais e de como cada indivíduo percebe a sua QV. Trata-se de um componente essencial para a saúde, uma vez que é permeada por indicadores que fogem da esfera biológica. Conforme definição da Organização Mundial da Saúde, qualidade de vida é "a percepção do indivíduo de sua inserção na vida, no contexto da cultura e sistemas de valores, nos quais ele vive e em relação aos seus objetivos, expectativas, padrões e preocupações"(7).

Pesquisas acerca da QV têm sido cada vez mais frequentes e indispensáveis no meio acadêmico, em especial, nos cursos da área da saúde, principalmente da enfermagem, porém com produção ainda incipiente quando se trata da área técnica. Para mensurar a QV, existem muitos instrumentos de verificação que propiciam a avaliação mais completa do seu impacto no cotidiano da vida dos investigados, como é o caso do WHOQOL-bref ${ }^{(8)}$.

Os instrumentos genéricos de avaliação da QV se aplicam às mais diferentes condições e refletem os diversos aspectos da vida das pessoas. Essa diversidade de aspectos se organiza em conjuntos, chamados de dimensões ou domínios, que são medidas de forma individualizada e 
ponderada $^{(8)}$ e são importantes para a compreensão dos fatores intervenientes na QV da população.

Nesse sentido, independentemente de o distanciamento social ser uma estratégia importante para a conservação da saúde física do indivíduo, é fundamental pensar em seus impactos na saúde e na QV da população. Assim, compreendendo que as pessoas reagem e experienciam essa condição de forma diferenciada, a identificação do impacto da pandemia do SARS-CoV 2 na QV dos estudantes do curso técnico em enfermagem, profissionais em formação para o enfrentamento de condições de morbimortalidade, é importante para o planejamento pedagógico e para o desenvolvimento de ações de suporte emocional para o retorno das atividades presenciais.

Diante do exposto, o objetivo desta pesquisa é identificar o impacto na QV de estudantes do curso técnico em enfermagem quanto ao período de suspensão do calendário acadêmico pela pandemia do coronavírus SARSCoV-2.

\section{MÉTODO}

Estudo transversal, desenvolvido com estudantes de um curso técnico em enfermagem de uma instituição pública na cidade de CuritibaPR. No período de coleta de dados, havia 60 estudantes matriculados e somente cinco não aceitaram participar. Todos os estudantes foram convidados, portanto não houve cálculo amostral. Foram considerados como critérios de inclusão: ser estudante ingressante no curso técnico em enfermagem, nos anos de 2019 ou 2020, estar regularmente matriculado e ser maior de 18 anos e como critérios de exclusão: os participantes que deixaram de responder mais de três questões dos questionários aplicados.

A coleta de dados ocorreu, por meio de plataformas on-line pela suspensão do calendário acadêmico na instituição participante, desde março de 2020. As pesquisadoras entraram em contato com os participantes, por meio de seus emails pessoais, disponibilizados pela coordenação do curso e encaminharam mensagem convidandoos a participar da pesquisa e disponibilizando explicação sobre os objetivos, a metodologia utilizada e o link para preenchimento do questionário via Google forms ${ }^{\circ}$. Os dados foram coletados, no mês de julho de 2020, por meio de dois instrumentos: um questionário sociodemográfico, elaborado pelos pesquisadores, e o WHOQOL-bref ${ }^{(8)}$, instrumento validado e disponibilizado pela Organização Mundial da Saúde.

O questionário sociodemográfico foi composto por perguntas de múltipla escolha que abordavam características como: idade, sexo (masculino/feminino), situação conjugal (casado/com companheiro/em união estável ou solteiro), número de filhos (opções de zero até quatro ou mais), situação ocupacional (trabalho remunerado e modalidade remota ou presencial, não trabalha por desemprego ou por opção) e grau de contribuição na renda familiar (não contribui, contribui parcialmente ou é o único mantenedor).

O WHOQOL-bref ${ }^{(8)}$ foi aplicado para a investigação da QV dos discentes. É composto por 26 questões, duas das quais referindo-se à qualidade de vida geral e à satisfação com a saúde e as demais (24 questões) distribuídas entre os domínios: Físico, Psicológico, das Relações Sociais e do Meio Ambiente ${ }^{(8)}$.

Para a análise dos dados sociodemográficos, utilizou-se o software Stata ${ }^{\circledR}$ versão 12 (Stata Corp., College Station, Estados Unidos), mediante estatística descritiva. Foram utilizadas frequências relativas e absolutas como medidas descritivas para as variáveis categóricas e média e desvio-padrão para as variáveis numéricas.

Para a análise e tratamento de dados relacionados à QV, foram adotadas as seguintes avaliações: as duas questões gerais foram calculadas em conjunto, para gerar um escore único e independente dos demais domínios, que denominamos Índice Geral de Qualidade de Vida (IGQV) e, para a análise dos demais dados do WHOQOL-bref ${ }^{(8)}$, utilizou-se uma ferramenta construída por Pedroso et al. ${ }^{(9)}$, que realiza cálculos dos escores e estatística descritiva de forma automatizada, disponibilizada em formato.xIs. A lógica utilizada por essa ferramenta é a média aritmética simples dos escores das 26 questões do questionário - considerados seus quatro domínios, para convertê-las em uma escala de 0 a 100 e exibi-las em gráficos. 0 escore de cada domínio é obtido em uma escala positiva, isto é, quanto mais alto o escore, melhor a QV naquele domínio.

Após a análise descritiva, foi adotada estatística não paramétrica de cada domínio e do IGQV para cada variável sociodemográfica, para verificar se haviam diferenças significativas entre as categorias, visto que as variáveis numéricas não 
tiveram aderência à distribuição normal. Para tal, foi utilizado o teste de Mann-Whitney para variáveis com duas categorias e Kruskal-Wallis para três ou mais categorias, considerando significativo o valor de $p<0,05$.

A pesquisa foi aprovada por Comitê de Ética em Pesquisa sob o N.o CAAE 20712719.7.0000.8156, em 17 de junho de 2020.

\section{RESULTADOS E DISCUSSÃO}

Participaram da pesquisa 55 estudantes. A idade média foi de $26,26 \pm 8,18$ anos, com mínima de 18 e máxima de 51 anos. Os demais dados sociodemográficos de caracterização dos participantes são apresentados na Tabela 1.

Tabela 1 - Dados sociodemográficos de caracterização dos participantes. Brasil, PR, Curitiba, 2020.

\begin{tabular}{lcc}
\hline \hline Variável & $\mathbf{n}$ & $\%$ \\
\hline & & \\
Sexo & 40 & $72,7 \%$ \\
$\quad$ Feminino & 15 & $27,3 \%$ \\
Masculino & \\
Situação conjugal & 29 & $52,7 \%$ \\
Solteiro & $26,3 \%$ \\
Casado/união estável/com companheiro & $47,3 \%$ \\
Número de filhos & 39 \\
Nenhum & 4 & $70,9 \%$ \\
Um & 7 & $7,3 \%$ \\
Dois & $12,7 \%$ \\
Três & 5 & $9,09 \%$ \\
Você está trabalhando de forma remunerada atualmente? & 17 \\
Sim, trabalho exclusivamente de forma presencial & 17 \\
Já estava desempregado antes da pandemia de COVID-19 & $30,9 \%$ \\
Não estou trabalhando no momento por opção própria & $30,9 \%$ \\
Sim, trabalho exclusivamente de forma remota & $16,4 \%$ \\
Perdi o emprego em razão da pandemia de COVID-19 & 4 \\
Sim, trabalho de forma alternada entre presencial e remoto & $7,3 \%$ \\
Não respondeu & 4 & $7,3 \%$ \\
Você é responsável economicamente pelo sustento da sua casa? & 3 & $5,5 \%$ \\
Não sou responsável pelo sustento da casa & 1 & $1,8 \%$ \\
Sim, minha renda complementa o sustento da casa & \\
Sim, sou o único responsável pelo sustento da casa & 30 \\
\hline \hline
\end{tabular}

Fonte: elaboração própria.

O perfil dos estudantes do curso técnico em enfermagem pesquisados demonstrou prevalência de mulheres. Dados semelhantes aos evidenciados foram encontrados em estudo desenvolvido, em duas Instituições Federais de Goiás, cujo objetivo foi investigar a qualidade de vida de estudantes do curso técnico em enfermagem e os desafios associados à formação profissional ${ }^{(10)}$. Resultados similares também advêm de outros estudos com o mesmo perfil de participantes $^{(11-13)}$, os quais evidenciaram 0 predomínio de participantes do sexo feminino em estudos envolvendo profissionais da enfermagem, que pode ser justificado pelo fato de a profissão ser predominantemente constituída por mulheres $^{(14)}$, o que denota que o cuidado ainda tem sido uma atividade relacionada ao exercício feminino.

Ainda sobre o perfil dos participantes, observa-se a prevalência de pessoas solteiras, sem filhos e com vínculo empregatício, resultados corroborados por estudo desenvolvido entre acadêmicos de enfermagem do Piauí(12). Esses dados refletem a situação atual no cenário mundial, cuja decisão pela constituição familiar, incluindo o casamento e a parentalidade, entre outras escolhas, tem sido adiada em função da realização profissional e da estabilidade financeira ${ }^{(15)}$.

No que diz respeito ao IGQV, a autoavaliação dos participantes foi de $68,9 \%$. Apesar do momento pandêmico vivenciado, os resultados apresentados neste estudo se assemelham a outros envolvendo acadêmicos da área da saúde ${ }^{(16-17)}$, cujos índices foram de $65,3 \%$ e $68,4 \%$, considerados bons, de acordo com escala da Organização Mundial da Saúde, que classifica zero (0) como a pior percepção de QV e cem (100) a melhor ${ }^{(9)}$. 
A maioria dos participantes avalia sua QV e sua saúde como boa, conforme demonstrado na Figura 1. Esses resultados divergem dos obtidos em pesquisa realizada entre técnicos de enfermagem ${ }^{(10)}$ cujos escores foram de $35,8 \%$ e $30,9 \%$, respectivamente.

Figura 1 - Avaliação dos estudantes do curso técnico em enfermagem sobre sua qualidade de vida e satisfação com a saúde. Brasil, PR, Curitiba, 2020.

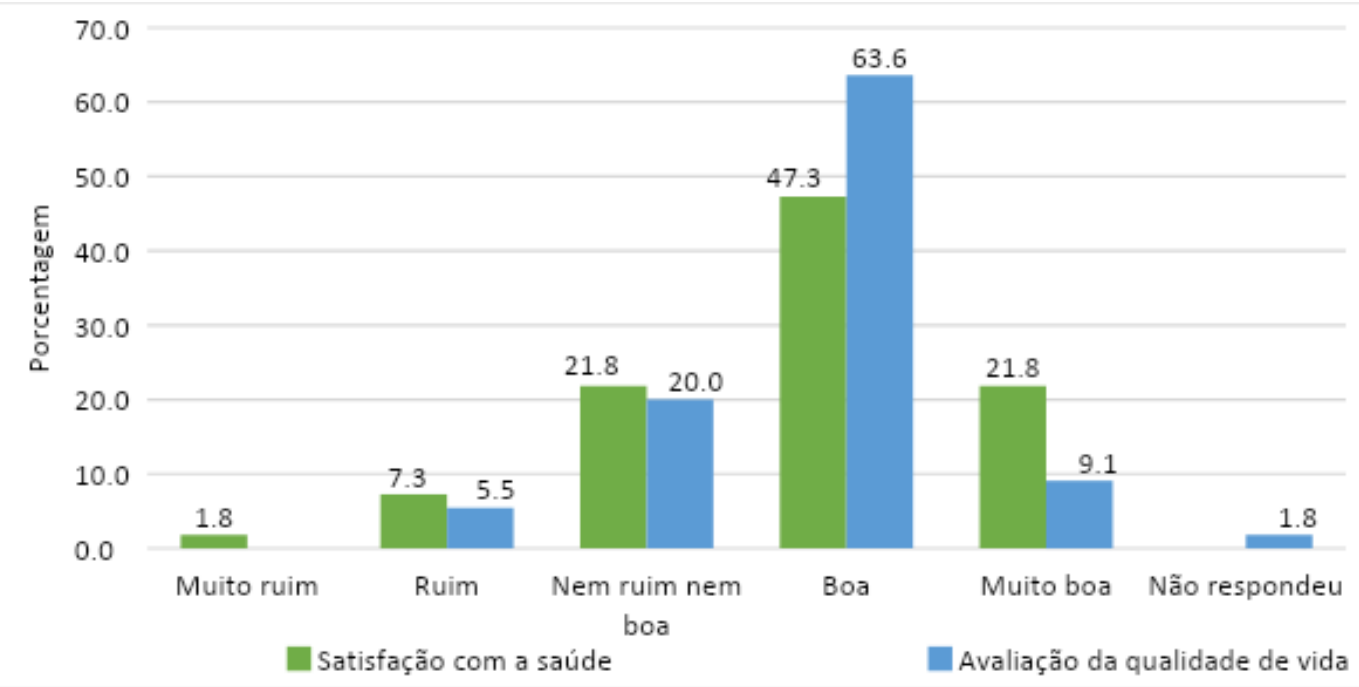

Fonte: elaboração própria.

Esses dados talvez se justifiquem pela suspensão do calendário acadêmico na instituição que foi cenário da pesquisa, o que, de certa forma, reduz a sobrecarga de tarefas, ou pela não percepção até este momento acerca dos efeitos das medidas sanitárias em suas rotinas diárias. De acordo com pesquisa sobre a temática ${ }^{(18)}$, quando avaliada no contexto acadêmico, a baixa Qualidade de Vida entre discentes de enfermagem está relacionada às exigências acadêmicas, à realização de aulas práticas em ambientes de saúde, à elaboração de trabalhos de conclusão de curso, entre outras demandas pedagógicas. Ademais, o curto período de tempo entre o início da pandemia e a coleta de dados pode não ter sido suficiente, para a manifestação de sintomas psicológicos, o que geralmente ocorre em um maior espaço de tempo.

Por outro lado, a participação em atividades sociais, realização de atividades de lazer e a discussão de problemas pessoais com amigos e familiares são preditoras de melhor $\mathrm{QV}^{(19)}$, situações incondizentes com o distanciamento social imposto à população por ocasião da COVID19 , porém compatíveis com o escore do domínio das Relações Sociais obtido no estudo (63,8\%).

Apesar das medidas sanitárias de distanciamento social, o domínio das Relações Sociais foi um dos que obteve o maior escore, o que incita uma melhor avaliação para a adequada compreensão desse resultado, a fim de identificar se é referente à não obediência às orientações de distanciamento social ou se novas estratégias de interação social foram desenvolvidas. Ademais, faz-se necessária uma investigação sobre os fatores associados à QV, neste público específico, bem como quanto à adesão às recomendações de distanciamento para melhor compreensão dos resultados obtidos.

Ao se avaliar a contribuição dos diferentes domínios para a QV dos discentes, observou-se que o domínio físico contribuiu positivamente, $67,9 \%$, assim como o domínio das relações sociais, $63,8 \%$. Por outro lado, os domínios do meio ambiente $(59,3 \%)$ e psicológico $(58,2 \%)$ obtiveram os menores escores, o que significa que tiveram a menor contribuição para o IGQV. Estes resultados se assemelham a outras pesquisas sobre a temática ${ }^{(10,12)}$, fato que chama a atenção na população pesquisada, neste período de pandemia, pois o distanciamento social tem sido uma das práticas recomendadas pela ciência e tem sido uma das orientações da Secretaria de Saúde, mantida até a atualidade no município de Curitiba. Por meio dessa prática, que busca restringir a circulação das pessoas com o objetivo de diminuir a circulação do vírus e, consequentemente, reduzir $o$ número de infectados $^{(1)}$, esperava-se que os domínios físico e das relações sociais fossem avaliados com menores escores, visto a limitação nas atividades sociais, realização de atividades físicas, além do 
impacto psicológico, comum em situações de risco de adoecimento, além dos impactos financeiros.

O Gráfico 2 apresenta o escore dos alunos do curso técnico em enfermagem em cada uma das facetas do WHOQOL-bref ${ }^{(8)}$. A faceta que obteve maior escore foi "Mobilidade" $(90,4 \%)$, enquanto a que teve menor escore foi a de "Recursos financeiros" (46,4\%).

Figura 2 - Escore dos estudantes do curso técnico em enfermagem nas diferentes facetas do WHOQOLbref $^{(8)}$. Brasil, PR, Curitiba, 2020.

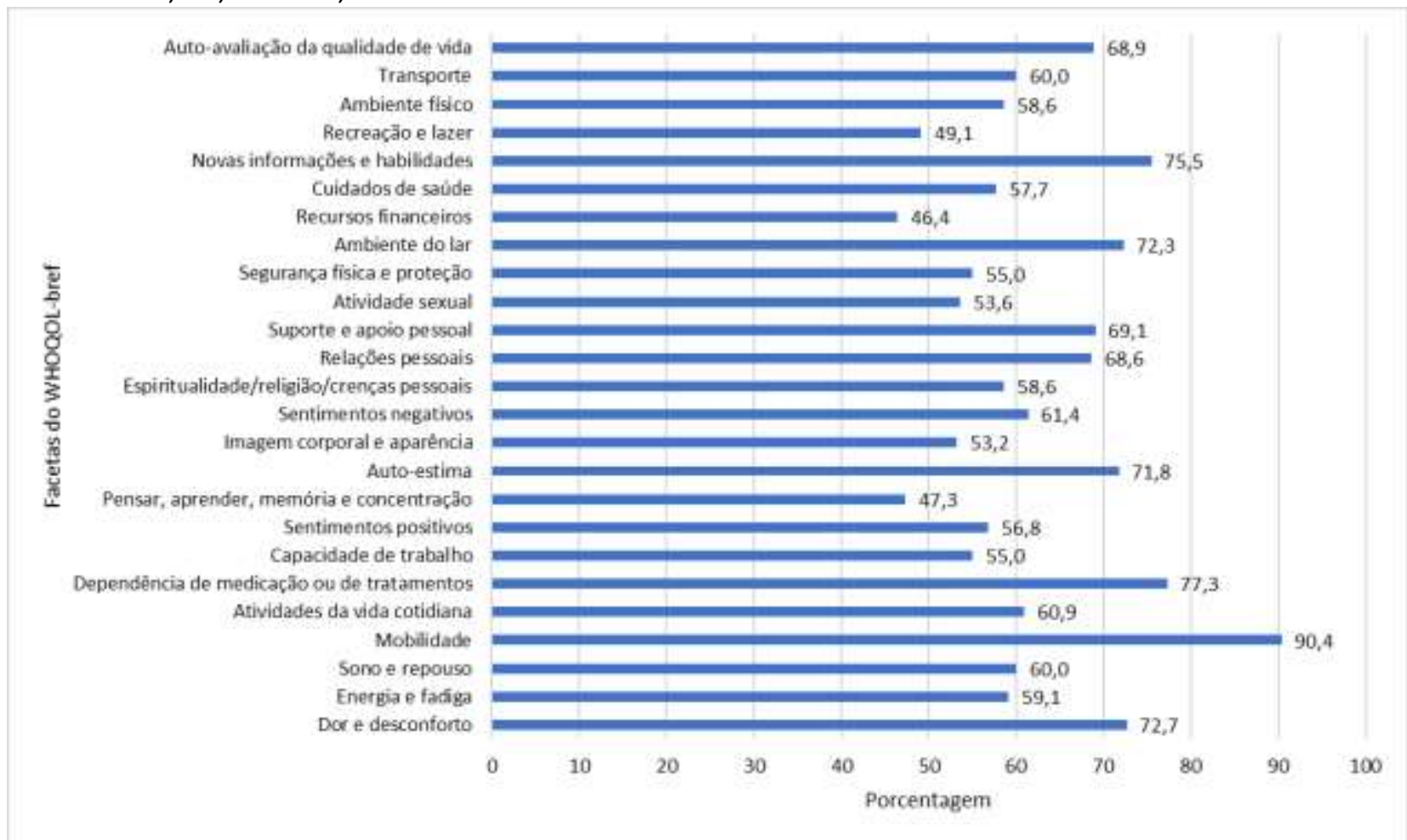

Fonte: elaboração própria.

Neste período pandêmico, tem sido evidenciado que a prevalência de Transtornos Mentais Comuns (TMC) encontram-se em franca ascendência ${ }^{(20)}$. No presente estudo, a faceta dos sentimentos negativos, cujo percentual foi de $61,4 \%$, denota a frequência com que o participante se sente mal-humorado e/ou triste, indicando um risco para a ocorrência de TMC. Estudo desenvolvido, para avaliar a qualidade de vida de 42 alunos de um curso de graduação em enfermagem, também, encontrou resultados semelhantes ${ }^{(21)}$. Apesar de sentimentos negativos serem evidenciados em estudos, em outros contextos sociais, no momento atual, isso pode estar mais perceptível, pois a população e os estudantes não conseguem se desvencilhar do tema e, por conseguinte, do sofrimento, como evidenciado também em estudo Chinês ${ }^{(4)}$, cuja população vivenciou o início da pandemia.

Outra faceta que chama atenção é a relacionada ao pensar, aprender, memória e concentração, cujo percentual foi de $47,3 \%$, o que denota a frequência com que o participante se recorda do que aprendeu e se sente cansado ou estressado ao se concentrar em alguma atividade. Esse resultado indica que a maioria dos participantes não consegue realizar ações essenciais para o aprendizado de novos conteúdos e habilidades, fato relevante quando se fala em estudantes. Destaca-se que os discentes pesquisados não estavam participando de aulas remotas previamente ou durante a coleta de dados, contudo incita-se sobre a importância de se considerar esse achado nas discussões sobre a oferta de atividades remotas, em especial, na área da saúde. No mais, essa faceta pode ainda estar relacionada à satisfação com o sono e repouso, pertencente ao domínio físico, a qual obteve percentual de $60 \%$.

Na Tabela 2 são apresentados os percentis de QV para cada um dos domínios do WHOQOLbref $^{(8)}$. No tocante à idade, os participantes foram categorizados em tercis, a saber: 1 o tercil -18 a 20 anos $(34,55 \%, \mathrm{n}=19) ; 20$ tercil -21 a 28 anos $(36,36 \%, \mathrm{n}=20)$; 3o tercil -29 a 51 anos $(29,09 \%$, $\mathrm{n}=19)$. 
Tabela 2 - Domínios do WHOQOL-bref ${ }^{(8)}$ de acordo com a variável idade. Brasil, PR, Curitiba, 2020.

\begin{tabular}{|c|c|c|c|c|c|c|c|}
\hline \multirow[t]{4}{*}{ Domínio } & \multirow{2}{*}{\multicolumn{6}{|c|}{$\begin{array}{c}\text { Variável } \\
\text { Idade }\end{array}$}} & \multirow[t]{4}{*}{ p-valor* } \\
\hline & & & & & & & \\
\hline & \multicolumn{2}{|c|}{10 tercil $(n=19)$} & \multicolumn{2}{|c|}{20 tercil $(n=20)$} & \multicolumn{2}{|c|}{ 3o tercil $(n=19)$} & \\
\hline & Mediana & $25-75 \%$ & Mediana & $25-75 \%$ & Mediana & $25-75 \%$ & \\
\hline Físico & 14,3 & $13,1-15,4$ & 16,0 & $12,6-16,6$ & 15,1 & $13,4-16,9$ & 0,5528 \\
\hline Psicológico & 13,3 & $11,3-15,3$ & 13,7 & $10,3-16,0$ & 14,0 & $13,0-15,3$ & 0,7129 \\
\hline Relações sociais & 12,0 & $10,7-16,0$ & 15,3 & $12,7-17,3$ & 14,7 & $12,7-16,0$ & 0,3260 \\
\hline Meio ambiente & 13,0 & $12,0-15,0$ & 13,8 & $11,8-15,3$ & 13,3 & $12,3-14,3$ & 0,7661 \\
\hline Autoavaliação da QV & 14,0 & $14,0-16,0$ & 16,0 & $14,0-16,0$ & 16,0 & $15,0-18,0$ & 0,0942 \\
\hline
\end{tabular}

Fonte: elaboração própria.

*Teste Kruskal-Wallis.

Para as demais variáveis sociodemográficas, foram utilizados quartis para a categorização e avaliação de cada um dos domínios do WHOQOLbref $^{(8)}$ (Tabela 3).

Tabela 3 - Domínios do WHOQOL-bref $f^{(8)}$ de acordo com variáveis sociodemográficas. Brasil, PR, Curitiba, 2020.

\begin{tabular}{|c|c|c|c|c|c|}
\hline \multirow[t]{2}{*}{ Domínio } & \multicolumn{4}{|c|}{ Variável } & \multirow[t]{2}{*}{ p-valor } \\
\hline & Mediana & $25-75 \%$ & Median & $25-75 \%$ & \\
\hline & \multicolumn{4}{|c|}{ Sexo } & \\
\hline & \multicolumn{2}{|c|}{ Feminino $(n=40)$} & \multicolumn{2}{|c|}{ Masculino ( $n=15)$} & \\
\hline Físico & 14,9 & $13,1-16,6$ & 15,4 & $13,7-17,1$ & $0,3677^{ \pm}$ \\
\hline Psicológico & 13,7 & $11,3-15,3$ & 14,7 & $10,0-17,3$ & $0,4146^{ \pm}$ \\
\hline Relações sociais & 14,7 & $12,0-16,0$ & 14,7 & $10,7-17,3$ & $0,9619^{ \pm}$ \\
\hline Meio ambiente & 13,0 & $12,0-14,5$ & 14,5 & $12,5-15,0$ & $0,3018^{ \pm}$ \\
\hline \multirow[t]{3}{*}{ Autoavaliação da QV } & 16,0 & $14,0-16,0$ & 16,0 & $14,0-16,0$ & $0,6299^{ \pm}$ \\
\hline & \multicolumn{4}{|c|}{ Situação conjugal } & \\
\hline & \multicolumn{2}{|c|}{ Solteiro $(n=29)$} & \multicolumn{2}{|c|}{ Com companheiro $(n=26)$} & \\
\hline Físico & 14,3 & $13,1-16,0$ & 16,0 & $13,7-16,0$ & $0,1217^{*}$ \\
\hline Psicológico & 12,7 & $10,0-14,7$ & 14,3 & $13,3-16,0$ & $0,0344^{*}$ \\
\hline Relações sociais & 12,0 & $10,7-16,0$ & 16,0 & $13,3-16,0$ & $0,0541^{*}$ \\
\hline Meio ambiente & 13,0 & $12,0-15,0$ & 13,5 & $12,0-14,5$ & $0,9663^{*}$ \\
\hline \multirow[t]{3}{*}{ Autoavaliação da QV } & 14,0 & $14,0-16,0$ & 16,0 & $16,0-18,0$ & $0,0025^{*}$ \\
\hline & \multicolumn{4}{|c|}{ Tem filhos? } & \\
\hline & \multicolumn{2}{|c|}{ Não (n = 39) } & \multicolumn{2}{|c|}{$\operatorname{Sim}(n=16)$} & \\
\hline Físico & 14,9 & $13,1-16,0$ & 16,6 & $13,7-17,1$ & $0,1119^{ \pm}$ \\
\hline Psicológico & 13,3 & $10,0-15,3$ & 14,0 & $13,0-16,0$ & $0,3719^{ \pm}$ \\
\hline Relações sociais & 13,3 & $10,7-16,0$ & 15,3 & $13,3-16,0$ & $0,3536^{ \pm}$ \\
\hline Meio ambiente & 13,5 & $12,0-15,5$ & 13,3 & $12,0-14,0$ & $0,4407^{ \pm}$ \\
\hline \multirow[t]{3}{*}{ Autoavaliação da QV } & 16,0 & $14,0-16,0$ & 14,5 & $13,2-15,5$ & $0,0212^{ \pm}$ \\
\hline & \multicolumn{4}{|c|}{ Está trabalhando no momento? } & \\
\hline & \multicolumn{2}{|c|}{$\operatorname{Sim}(n=24)$} & \multicolumn{2}{|c|}{ Não $(n=30)$} & \\
\hline Físico & 14,9 & $13,4-16,9$ & 14,9 & $13,1-16,6$ & $0,6624^{ \pm}$ \\
\hline Psicológico & 14,7 & $12,7-16,3$ & 13,3 & $10,0-14,7$ & $0,0392^{ \pm}$ \\
\hline Relações sociais & 15,3 & $12,7-18,0$ & 13,3 & $10,7-16,0$ & $0,0564^{ \pm}$ \\
\hline Meio ambiente & 13,8 & $12,3-15,5$ & 13,0 & $12,0-14,5$ & $0,3876^{ \pm}$ \\
\hline \multirow[t]{3}{*}{ Autoavaliação da QV } & 16,0 & $13,0-18,0$ & 16,0 & $14,0-16,0$ & $0,6841^{ \pm}$ \\
\hline & \multicolumn{4}{|c|}{ É responsável pelo sustento financeiro da casa? } & \\
\hline & \multicolumn{2}{|c|}{$\operatorname{Sim}(n=25)$} & \multicolumn{2}{|c|}{ Não $(n=30)$} & \\
\hline Físico & 16,0 & $13,7-17,1$ & 14,9 & $13,1-16,6$ & $0,1643^{ \pm}$ \\
\hline Psicológico & 14,0 & $13,3-16,0$ & 13,0 & $10,0-14,7$ & $0,0196^{ \pm}$ \\
\hline Relações sociais & 14,7 & $13,3-16,0$ & 13,3 & $10,7-16,0$ & $0,1325^{ \pm}$ \\
\hline Meio ambiente & 13,0 & $12,0-14,5$ & 13,5 & $12,0-15,0$ & $0,8922^{ \pm}$ \\
\hline Autoavaliação da QV & 16,0 & $14,0-18,0$ & 16,0 & $14,0-16,0$ & $0,3887^{ \pm}$ \\
\hline
\end{tabular}

Fonte: elaboração própria.

*Teste Kruskal Wallis; ${ }^{ \pm}$Teste Mann Whitney.

Nesse estudo observou-se que as variáveis relacionadas à situação conjugal, trabalho e sustento da casa alteram de forma significativa a qualidade de vida, principalmente, no que diz respeito à dimensão psicológica, o que coaduna com outros estudos que também identificaram 
associação entre distúrbios psicológicos (depressão), renda familiar ${ }^{(3,21)}$ e situação conjugal $^{(3)}$. De acordo com estudos recentes, os impactos psicológicos surgem, a partir de situações como a disseminação rápida do vírus por todo o mundo, as dificuldades de controle e tratamento, a imprevisibilidade relacionada à duração da pandemia e seus desdobramentos ${ }^{(22)}$, o risco de adoecimento de familiares, bem como o desconhecimento sobre as medidas de prevenção e controle, entre outros ${ }^{(4)}$.

Os achados deste estudo concordam com outros estudos realizados até o momento, relacionados à saúde mental e aos impactos causados pela pandemia, como o realizado entre 619 estudantes universitários, cujo objetivo foi analisar se os níveis de depressão, ansiedade e estresse se alteraram no período pandêmico, comparativamente com períodos anteriores. Os resultados confirmaram um aumento significativo de perturbação psicológica (ansiedade, depressão e estresse $)^{(2)}$.

Também se identificou que as variáveis situação conjugal e trabalho impactam na dimensão das relações sociais, as quais se encontram relacionadas a uma das facetas importantes na avaliação da QV, a do suporte e apoio pessoal, cujo índice foi de $69,1 \%$. Esse resultado corrobora com os achados de estudo desenvolvido entre estudantes de medicina da Turquia $^{(3)}$, o qual identificou que a situação conjugal e o trabalho atuam como fatores de proteção à saúde, uma vez que reduzem os efeitos da pandemia, acrescentando que aqueles que moravam com a família também sentiram menores efeitos.

$E$, ainda, diante da autoavaliação da QV, as variáveis que determinaram significativo impacto foram a situação conjugal e filhos, pois a presença do companheiro para dividir suas angústias e/ou atividades cotidianas influencia positivamente na QV, bem como a ausência de filhos, o que demandaria o emprego de energia física, psicológica e financeira, conforme evidenciado também em outros estudos ${ }^{(4,21)}$.

Verificou-se que $\mathrm{o}$ período pandêmico interferiu na QV da população estudada, sobretudo, no que se refere aos aspectos psicológicos já discutidos. Suscita-se uma problemática a ser referenciada pelas instituições de ensino, em projetos futuros de auxílio a esses estudantes, quando houver o retorno às aulas presenciais, bem como no desenvolvimento de estratégias de proteção à saúde mental e melhoria da QV durante o ensino remoto.

$O$ fato de as desordens psicológicas terem uma evolução lenta e as manifestações ocorrerem de forma prolongada e gradual, podendo ser evidenciada somente anos após o término do período pandêmico ${ }^{(23)}$, esses resultados podem ainda não estar refletindo o real impacto do isolamento social neste público. No entanto já fornecem subsídios para o planejamento de ações em curto e longo prazo, no âmbito das instituições de ensino, de modo que minimizem as possíveis consequências para o aprendizado discente, para a atuação docente e, consequente interferência na formação e atuação profissional.

\section{CONCLUSÃO}

O estudo evidenciou que o momento pandêmico exerceu influência na qualidade de vida dos estudantes do curso técnico de enfermagem durante o período de suspensão do calendário acadêmico. Apesar da autoavaliação positiva para a QV e para a saúde geral, a dimensão psicológica obteve o menor escore na avaliação dos domínios. Além disso, identificou-se que as variáveis relacionadas à situação conjugal, filhos, trabalho e sustento da casa alteram de forma significativa a qualidade de vida dos estudantes investigados durante a pandemia.

Conhecer o impacto da pandemia, na qualidade de vida dos estudantes do curso técnico de enfermagem, pode contribuir com as instituições formadoras no sentido de desenvolver estratégias que minimizem os seus efeitos na saúde e na QV dessa população, como, por exemplo, a implementação de programas de acompanhamento psicológico que poderá contribuir para a prevenção de patologias de ordem psicológica e auxiliar no enfrentamento das angústias, no retorno às atividades presenciais no curso, bem como no retorno às suas rotinas diárias de vida.

Entre as limitações deste estudo, destaca-se a amostragem limitada, o que dificulta a generalização dos resultados, bem como a realização de análises mais robustas. No mais, há escassez de estudos que avaliam a qualidade de vida especificamente de estudantes do curso técnico em enfermagem, fato que dificulta a discussão dos dados. Outras características dos estudos transversais são as limitações atribuídas ao potencial viés de memória e a transversalidade reversa. Apesar das limitações, acredita-se que a 
garantia do anonimato possa ter reduzido o viés de informação.

\section{REFERÊNCIAS}

1 - Wilder-Smith A, Freedman DO. Isolation, quarantine, social distancing and community containment: Pivotal role for old-style public health measures in the novel coronavirus (2019nCoV) outbreak. J Travel Med. 2020;27(2):taaa020. DOI: 10.1093/itm/taaa020 2 - Maia BR, Dias PC. Ansiedade, depressão e estresse em estudantes universitários: $O$ impacto da COVID-19. Estud Psicol. 2020;37:e200067. DOI: 10.1590/1982-0275202037e200067

3 - Torun F, Torun SD. O impacto psicológico da pandemia COVID-19 em estudantes de medicina na Turquia. Pak J Med Sci. 2020;36(6):1355-9. DOI: $10.12669 /$ pims.36.6.2985

4 - Wang C, Pan R, Wan X, Tan Y, Xu L, Ho CS, et al. Immediate psychological responses and associated factors during the initial stage of the 2019 Coronavirus Disease (COVID-19) epidemic among the general population in China. Int J Environ Res Public Health 2020;17:1729. DOI: 10.3390/ijerph17051729

5 - Vommaro P. O mundo em tempos de pandemia: Certezas, dilemas e perspectivas. Rev Direito Práxis Ahead of print 2020;(10):1-20. DOI: 10.1590/2179-8966/2020/51001

6 -Leigh J, Vasilica C, Dron R, Gawthorpe D, Burns $E$, Kennedy $S$, et al. Redefining undergraduate nurse teaching during the coronavirus pandemic: Use of digital technologies. $\mathrm{Br} J$ Nurs. 2020;29(10):566-9.

DOI: 10.12968/bjon.2020.29.10.566

7 - Organização Mundial de Saúde (OMS). Promoción de la salud: Glosario. Ginebra: Organización Mundial de la Salud; 1998.

8 - Fleck MPA, Louzada S, Xavier M, Chachamovich E, Vieira G, Santos L, et al. Application of the portuguese version of the abbreviated instrument of quality life WHOQOL-bref. Rev Saúde Pública 2000;34(2):178-83. DOI: 10.1590/s003489102000000200012

9 - Pedroso B, Pilatti LA, Gutierrez GL, Picinin CT. Cálculo dos escores e estatística descritiva do WHOQOL-bref através do Microsoft Excel. Rev Bras Qual Vida 2010;2(1):31-6. DOI:10.3895/S2175-08582010000100004

10 - Nunes PS, Marinho TA, Campanati FLS, Silva NP, Pedroso CF, Nóbrega MM, et al. Qualidade de vida de estudantes do curso técnico em enfermagem. Rev Enferm UFPE 2019;13:e242601. DOI: $\underline{10.1590 / S 0080-62342008000100008}$
11 - Oliveira AAC. Evasão de um curso técnico de enfermagem: Percepção de estudantes não concluintes. [dissertação]. São Paulo: Pontifícia Universidade de São Paulo; 2016.

12 - Moura IH, Nobre RS, Cortez RMA, Campelo V, Macêdo SF, Silva ARV. Qualidade de vida de estudantes de graduação em enfermagem. Rev Gaúcha Enferm. 2016;37(2):e55291. DOI: 10.1590/1983-1447.2016.02.55291

13 - Chaves ECL, lunes DH, Moura CC, Carvalho LC, Silva AM, Carvalho EC. Anxiety and spirituality in university students: A cross-sectional study. Re Bras Enferm. 2015;68(3):444-9. DOI: 10.1590/0034-7167.2015680318i

14 - Machado MH, coordenador. Perfil da enfermagem no Brasil: Relatório final. Rio de Janeiro: Fiocruz; 2017.

15 - Bernardi D, Féres-Carneiro T, Magalhães AS. Entre o desejo e a decisão: A escolha por ter filhos na atualidade. Contextos Clínic 2018;11(2):16173. DOI: $10.4013 /$ ctc.2018.112.02

16 - Anversa AC, Santos Filha VAV, Silva EB, Fedosse E. Qualidade de vida e o cotidiano acadêmico: Uma reflexão necessária. Cad Bras Ter Ocup. 2018;26(3):626-31. DOI: 10.4322/25268910.ctoAO1185

17 - Salgado RDC, Siqueira SS, Salgado TC. Qualidade de vida do estudante trabalhador: Uma amostra dos discentes de cursos superiores do Instituto Federal do Piauí - Campus Floriano. Rev Somma 2016 [citado em: 06 ago 2020]; 2(2):35-46. Acesso em:

http://ojs.ifpi.edu.br/revistas/index.php/somma 18 - Pereira MO, Pinho PH, Cortes JM. Qualidade de vida: Percepção de discentes de graduação em enfermagem. J Nurs Health 2016;6(2):321-33. DOI: $10.15210 /$ JONAH.V6I2.5780

19 - ilhan N, Peker K, Yıldırım G, Baykut G, Bayraktar $M$, Yıldırım $H$. Relationship between healthy lifestyle behaviors and health related quality of life in Turkish school-going adolescents. Niger J Clin Pract. 2019;22:1742-51. DOI: 10.4103/njcp.njcp 19019

20 - Brooks SK, Webster RK, Smith LE, Woodland L, Wessely S, Greenberg N, et al. The psychological impact of quarantine and how to reduce it: Rapid review of the evidence. Lancet 2020;395(10227):912-20. DOI: 10.1016/S01406736(20)30460-8

21 - Pinheiro JMG, Macedo ABT, Antoniolli L, Dornelles TM, Tavares JP, Souza SBC. Qualidade de vida, sintomas depressivos e psiquiátricos menores em estudantes de enfermagem. Rev Bras 
Enferm. 2020;73(supl 1):e20190134. DOI: 10.1590/0034-7167-2019-0134

22 - Zandifar A, Badrfam R. Iranian mental health during the COVID-19 epidemic. Asian J Psychiatr 2020;51:101990. DOI: 10.1016/j.ajp.2020.101990 23 - Ornell F, Schuch JB, Sordi AO, Kessler FHP. "Pandemic fear" and COVID-19: Mental health burden and strategies. Braz J Psychiatry 2020;42(3):232-5. DOI: 10.1590/1516-4446-2020$\underline{0008}$

Nota: Não houve financiamento por agência de fomento.

Recebido em: 08/10/2020

Aprovado em: 17/12/2020

Endereço de correspondência:

Tangriane Hainiski Ramos

Rua João Negrão, 1285. Bairro Rebouças. Curitiba, Paraná,

Brasil. CEP: 80.230-150.

E-mail: tangriane.ramos@ifpr.edu.br 